\title{
Many-Body Decay of the Gapped Lowest Excitation of a Bose-Einstein Condensate
}

\author{
Jinyi Zhang® ${ }^{1,{ }^{*}}$ Christoph Eigen $\odot,{ }^{1, *,}$ Wei Zheng $\odot,{ }^{1,2,3, \ddagger}$ Jake A. P. Glidden $\odot,{ }^{1}$ Timon A. Hilker $\odot,{ }^{1}$ \\ Samuel J. Garratt $\odot,{ }^{1,4}$ Raphael Lopes $\odot,{ }^{1,5}$ Nigel R. Cooper $\odot,{ }^{1}$ Zoran Hadzibabic $\odot,{ }^{1}$ and Nir Navon $\oplus^{6}$ \\ ${ }^{1}$ Cavendish Laboratory, University of Cambridge, J. J. Thomson Avenue, Cambridge CB3 OHE, United Kingdom \\ ${ }^{2}$ Hefei National Laboratory for Physical Sciences at the Microscale and Department of Modern Physics, \\ University of Science and Technology of China, Hefei 230026, China \\ ${ }^{3}$ CAS Center for Excellence in Quantum Information and Quantum Physics, University of Science and Technology of China, \\ Hefei 230026, China \\ ${ }^{4}$ Theoretical Physics, University of Oxford, Parks Road, Oxford OX1 3PU, United Kingdom \\ ${ }^{5}$ Laboratoire Kastler Brossel, Collège de France, CNRS, ENS-PSL Research University, Sorbonne Université, \\ 11 Place Marcelin Berthelot, 75005 Paris, France \\ ${ }^{6}$ Department of Physics, Yale University, New Haven, Connecticut 06520, USA
}

(Received 23 April 2020; revised 9 December 2020; accepted 11 January 2021; published 12 February 2021)

\begin{abstract}
We study the decay mechanism of the gapped lowest-lying axial excitation of a quasipure atomic BoseEinstein condensate confined in a cylindrical box trap. Owing to the absence of accessible lower-energy modes, or direct coupling to an external bath, this excitation is protected against one-body (linear) decay, and the damping mechanism is exclusively nonlinear. We develop a universal theoretical model that explains this fundamentally nonlinear damping as a process whereby two quanta of the gapped lowest excitation mode couple to a higher-energy mode, which subsequently decays into a continuum. We find quantitative agreement between our experiments and the predictions of this model. Finally, by strongly driving the system below its (lowest) resonant frequency, we observe third-harmonic generation, a hallmark of nonlinear behavior.
\end{abstract}

DOI: 10.1103/PhysRevLett.126.060402

Much of our understanding of interacting quantum manybody systems is rooted in the existence of long-lived elementary excitations. The nature of these excitations reflects the form of order in the underlying state of matter. Moreover, the excitation energy spectrum gives access to the low-temperature thermodynamics $[1,2]$. To lowest order, it is typically calculated by assuming that the excitations are noninteracting and, thus, have an infinite lifetime.

In the continuum limit, taking into account the interactions between quasiparticles generically leads to finite lifetimes, even at zero temperature [3]. Such lifetimes play a crucial role in understanding transport properties of quantum fluids, such as their thermal and electrical conductivity, viscosity, or the attenuation of sound. The case of gapped excitations is profoundly different, owing to additional constraints on decay channels. Gaps in the energy spectra naturally arise in systems with discrete translational symmetry [4], or as a result of many-body [5-7] and finitesize effects [8,9]. The problem of the lifetime of gapped excitations has been considered in myriad contexts, including optical phonons in crystals [10], quasiparticles in quantum dots [11], magnons in spin systems [12,13], and rotons in superfluid helium [14-16]. However, the decay considered in these studies typically originated from the coupling to lower-lying modes (e.g., acoustic branches), or scattering due to thermally populated excitations.
In this Letter, we study the fundamental many-body problem of the lifetime of a gapped lowest-lying excitation at zero temperature. This situation is particularly intriguing since the excitation is energetically immune to any onebody (i.e., linear) decay. The finite lifetime exclusively arises from nonlinear effects and provides a window into the interactions between quasiparticles. We experimentally and theoretically study this problem in the textbook setting of a homogeneous box-trapped atomic Bose-Einstein condensate (BEC).

The weakly interacting bulk Bose gas has been a remarkable test bed for exploring the physics of excitations and their interactions [17-44]. At low temperature, the excitations of an infinite uniform BEC have an energy given by the Bogoliubov spectrum; in the long-wavelength limit, these excitations are phonons [47]. The leading decay channel for the phonons is a linear process, in which they spontaneously break up into pairs of longer-wavelength phonons [Fig. 1(a)], a damping mechanism first predicted by Beliaev [48].

By contrast, in our case, the finite system size leads to experimentally resolvable gaps in the excitation spectrum, and recent works suggested that the damping of the lowest mode is fully nonlinear (within experimental precision) $[41,49]$. Here, we experimentally and theoretically elucidate the excitation lifetime of this mode by investigating the 
(a)

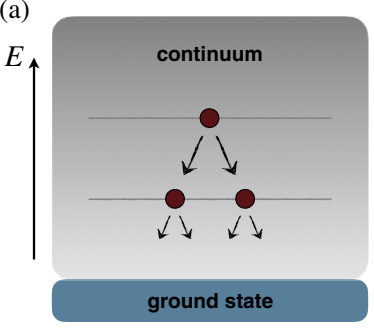

(b)

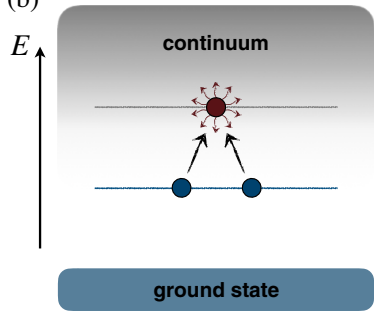

FIG. 1. Decay mechanisms of elementary excitations in a quantum many-body system. (a) Beliaev-like linear damping of an excitation in the continuum to two (or more) lower-lying excitations. (b) Nonlinear decay of the lowest-energy, gapped excitation (blue) to a higher-lying mode (red). The continuum is represented as a gray shade, while the horizontal lines indicate relevant energy levels.

steady-state response of the system to a continuous drive. We show that the underlying damping mechanism can be explained by a generic microscopic model based on an inverse Beliaev-like process, whereby two elementary excitations merge into a higher-energy one [Fig. 1(b)].

Our experiment starts with a quasiuniform BEC of ${ }^{87} \mathrm{Rb}$ atoms [50], prepared in a cylindrical optical box trap of radius $R=16(1) \mu \mathrm{m}$ and length $L=26(1) \mu \mathrm{m}$ [see Fig. 2(a)]. Our condensates consist of $N=1.2(1) \times 10^{5}$ atoms, their chemical potential is $\mu \approx k_{B} \times 2.0$ (3) nK, their healing length $\xi \approx 1.2(1) \mu \mathrm{m}$, and the excitation frequency of the lowest-lying axial mode [Fig. 2(b)] is $\omega_{d}=2 \pi \times 9.0(1) \mathrm{Hz}$ (see [41] for details). We excite this mode by applying a spatially uniform oscillating force $\mathbf{F}_{s}(\mathbf{r}, t)=\left(U_{s} / L\right) \sin (\omega t) \mathbf{e}_{z}$, where $\mathbf{e}_{z}$ is the unit vector along the symmetry axis of the cylinder and $U_{s}$ is the maximal potential-energy drop across the box. The force is produced by a pair of coils, which create a magnetic field gradient that couples to the magnetic moment of the atoms. Following a variable shaking time $t_{s}$, the BEC is released

and the center of mass of the atomic density distribution is recorded after a time of flight $t_{\mathrm{TOF}}=140 \mathrm{~ms}$, which reflects the in situ center-of-mass velocity $v\left(t_{s}\right)$ along $\mathbf{e}_{z}$.

In Fig. 2(c), we show examples of $v\left(t_{s}\right)$ for three drive frequencies (below, on, and above resonance), and for two drive amplitudes $\left(U_{s} / k_{B}=0.2\right.$ and $\left.1.6 \mathrm{nK}\right)$. While transient dynamics are visible at early times, a monochromatic steady state is established at later times. We fit the data for $t_{s}>0.6 \mathrm{~s}$ with $v\left(t_{s}\right)=v_{m} \sin \left(\omega t_{s}+\theta\right)$ (solid lines) and extract the amplitude $v_{m}$ and phase $\theta$ of the velocity response. For the weaker drive $\left(U_{s} / \mu \approx 0.1\right)$, we observe significant response only on resonance. However, for the stronger drive $\left(U_{s} / \mu \approx 0.8\right)$, we observe comparable response amplitudes at all three frequencies, indicating significant broadening of the response spectrum, a clear signature of nonlinear behavior. Specifically, such broadening corresponds to the shortening of the lifetime of excitations due to their mutual interactions.

To characterize this nonlinear behavior, we plot $v_{m}$ as a function of $U_{s}$ on log-log scale for various drive frequencies in Fig. 2(d). For linear response, with amplitudeindependent damping, one would get $v_{m} \propto U_{s}$, for all $\omega$. Instead, on resonance $\left(\omega=\omega_{d}\right)$, we observe power-law behavior $v_{m} \propto U_{s}^{0.32(1)}$, reminiscent of classical models with a cubic nonlinear damping (which generically predict $v_{m} \propto U_{s}^{1 / 3}$ on resonance; see Sec. I in [51]). Away from resonance, $v_{m}$ is smaller than on resonance (for the same $U_{s}$ ), but for stronger drives, a progressively broader range of frequencies becomes effectively resonant.

In the following, we introduce a theoretical model for this nonlinear behavior, and compare it to the full experimental response curves $v_{m}(\omega)$ and $\theta(\omega)$ (see Fig. 3). Within quantum theory, in the spirit of Fig. 1(b), we associate the cube-root scaling $v_{m} \propto U_{s}^{1 / 3}$ with the decay of the mode toward higher energies via a two-body process [52], resulting in a decay rate proportional to the number of
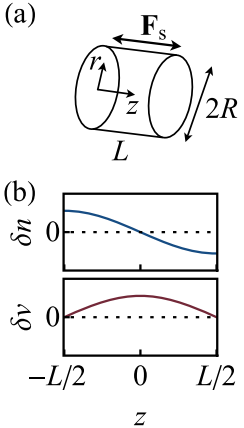

(c)

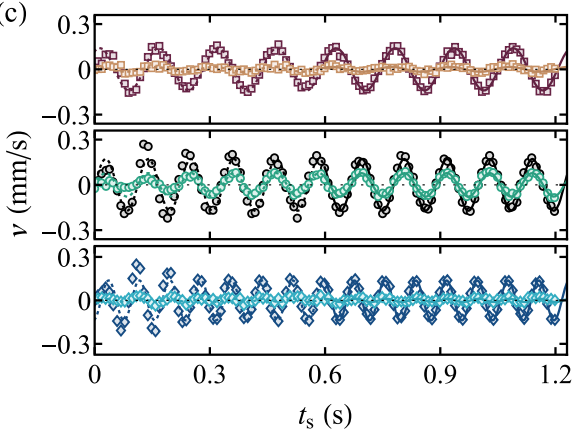

(d)

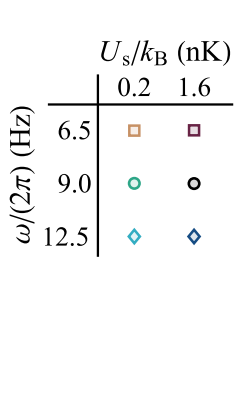

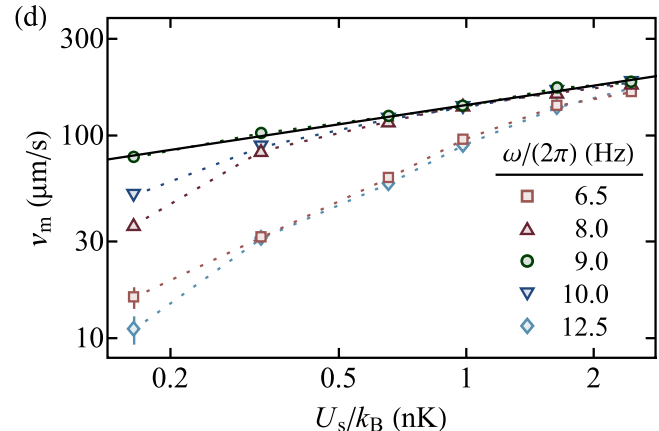

FIG. 2. Nonlinear dynamics of a driven box-trapped Bose-Einstein condensate. (a) Sketch of the optical box trap, highlighting the axis along which the drive $\mathbf{F}_{s}(t)$ is applied. (b) Sketch of the fundamental mode density $\delta n(z)$ and velocity $\delta v(z)$ profiles along the box axis. (c) Center-of-mass velocity response $v\left(t_{s}\right)$ for three different drive frequencies $\omega$, close to the resonant $\omega_{d} /(2 \pi)=9.0(1) \mathrm{Hz}$, and two different drive strengths $U_{s}$ (see legend). (d) Steady-state response amplitude $v_{m}$ versus $U_{s}$ on log-log scale, for various drive frequencies (see legend). The on-resonance $v_{m}$ exhibits power-law behavior, and a fit to the data (solid line) gives $v_{m} \propto U_{s}^{0.32(1)}$. For progressively larger $U_{s}$, a wider range of $\omega$ becomes effectively resonant. The dotted lines are guides to the eye, and the error bars represent fitting uncertainties. 

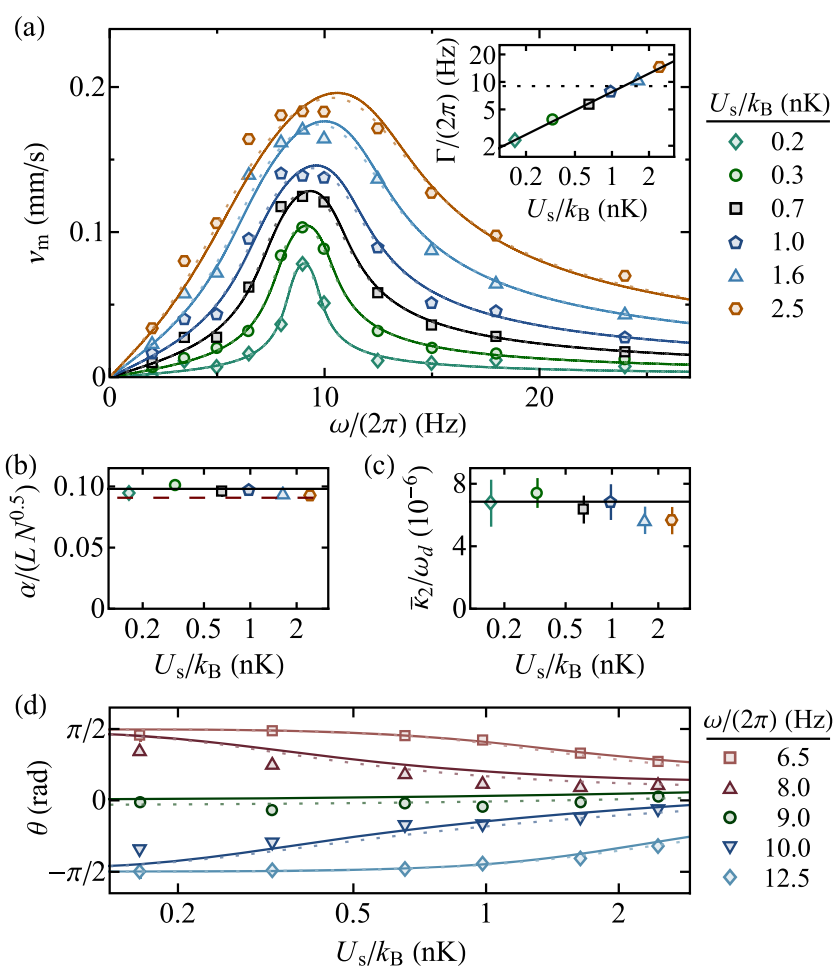

FIG. 3. Nonlinear response functions and comparison with theory. (a) Steady-state velocity response amplitude $v_{m}$ as a function of $\omega$, for six different drive strengths $U_{s}$ (see legend), alongside the corresponding fits to our model (see text for details). The inset shows $\Gamma$, the extracted full width at half maximum of $v_{m}(\omega)$, as a function of $U_{s}$ on log-log scale, while the horizontal dotted line indicates $\omega_{d}$. The solid line is a fit to the data, $\Gamma \propto U_{s}^{0.67(2)}$. For reference, our experimental upper bound on the linear damping rate is $2 \pi \times 0.3 \mathrm{~s}^{-1}$ [41]. (b),(c) Extracted model fit parameters for the response curves $v_{m}(\omega)$ in (a). The dashed line in (b) shows a theoretical estimate (see text), while the solid lines in (b) and (c) depict the average for $U_{s} / k_{B}<1 \mathrm{nK}$. (d) Phase response as a function of $U_{s}$. The solid lines show the prediction of the model with the extracted average parameters [solid lines in (b) and (c)] for $\operatorname{Re}\left[\kappa_{2}\right]=0$. The dotted lines, instead, show corresponding predictions which include a small nonzero $\operatorname{Re}\left[\kappa_{2}\right]$ (see text). Where not visible, the fitting error bars are smaller than the point size.

phonons. Once excited, this "secondary" mode couples to other modes, which act as a quasicontinuum of states to which the excitation can decay. We formalize this picture by developing a simple model in which the fundamental excitation of energy $\hbar \omega_{d}$ is created by an external forcing and is coupled to an auxiliary mode of energy $\hbar \omega_{b}$ (see Secs. II-VI in [51]). The Hamiltonian describing the system is

$$
\begin{aligned}
\hat{H}= & \hbar \omega_{d} \hat{d}^{\dagger} \hat{d}+\hbar \omega_{b} \hat{b}^{\dagger} \hat{b}+\hbar \lambda\left(\hat{b}^{\dagger} \hat{d} \hat{d}+\text { H.c. }\right) \\
& +\hbar \Omega \sin (\omega t)\left(\hat{d}^{\dagger}+\hat{d}\right),
\end{aligned}
$$

where $\hat{d}^{\dagger}(\hat{d})$ and $\hat{b}^{\dagger}(\hat{b})$ are, respectively, the creation (annihilation) operators for the fundamental and the auxiliary mode, $\lambda$ is the coupling strength, and $\Omega$ is the strength of the drive. We incorporate the decay of the auxiliary mode into the quasicontinuum via a master equation approach. By tracing out the auxiliary mode, we derive, within a mean-field approximation, an equation of motion for the mean dipole $d(t) \equiv\langle\hat{d}(t)\rangle$ :

$$
i \partial_{t} d-\left(\omega_{d}+\kappa_{2}|d|^{2}\right) d=\Omega \sin (\omega t) .
$$

The generally complex $\kappa_{2}$ captures the nonlinear effects to leading order. Specifically, $\operatorname{Re}\left[\kappa_{2}\right]$ and $\operatorname{Im}\left[\kappa_{2}\right]$ correspond, respectively, to a frequency shift (due to the selfinteraction) and a nonlinear damping (due to the mediated coupling to the continuum); expressions for $\kappa_{2}$ in terms of the microscopic model parameters are provided in Sec. III in [51]. In practice, $\kappa_{2}$ is sensitive to the details of the trapping potential, and it is more convenient to extract it directly from the experimental data. However, the form of Eq. (2) is universal in that it does not depend on the exact loss mechanism of the auxiliary mode, nor the number of auxiliary excitations involved in the elementary interaction process [see Eq. (1)].

To compare our experimental data to the theory, we relate $d(t)$ to the main experimental observable $v(t)=(2 \alpha / N) \partial_{t} \operatorname{Re}[d(t)]$, where $\alpha$ is the matrix element of the position operator $\hat{z}$ between the ground state and the lowest-lying excitation (see Sec. IV in [51]). In terms of the experimental parameters, the drive amplitude in Eq. (1) is $\Omega=\alpha U_{s} /(\hbar L)$.

We determine the parameters of the model by fitting the $v_{m}(\omega)$ response curves to the steady-state numerical solutions of Eq. (2) for each $U_{s}$. The resulting fits are shown in Fig. 3(a) as solid lines, where, for simplicity, we first neglect the real part of the nonlinear coefficient $\kappa_{2}$, so that the adjustable parameters are $\bar{\kappa}_{2} \equiv i \kappa_{2}$ and $\alpha$. We see that for $U_{s} \lesssim k_{B} \times 2 \mathrm{nK}$, the fitted model captures the experimental data well. Only for $U_{s} \gtrsim k_{B} \times 2 \mathrm{nK}$ do deviations between the model and the data become apparent. In the inset of Fig. 3(a), we plot the extracted full width at half maximum of the spectral lines, $\Gamma$, which is inversely proportional to the excitation lifetime, as a function of $U_{s}$. The plot reveals that the deviation between the model and the data occurs only once $\Gamma \gtrsim \omega_{d}$. For $U_{s} / k_{B}=1.6 \mathrm{nK}$, we estimate that $\bar{\kappa}_{2}|d|^{2} \approx 0.5 \omega_{d}$, and higher-order nonlinearities could become important.

The parameters extracted from each $v_{m}(\omega)$ curve are shown in Figs. 3(b) and 3(c). Crucially, both $\alpha$ and $\bar{\kappa}_{2}$ do not depend on $U_{s}$ within experimental errors, demonstrating that the model [Eq. (2)] captures the nonlinear $U_{s}$-dependent response. Averaging the fitted parameters within the range of validity of the model $\left(U_{s} / k_{B}<1 \mathrm{nK}\right)$, we obtain $\alpha /(L \sqrt{N})=0.098(4)$ and $\bar{\kappa}_{2} / \omega_{d}=6.9(7) \times 10^{-6}$ per phonon (solid horizontal lines). A calculation assuming a cylindrical-box-trapped BEC in the Thomas-Fermi regime (Sec. III in [51]) yields 

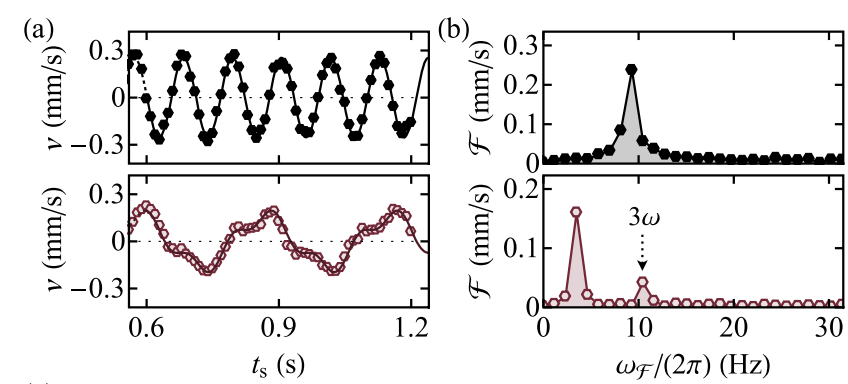

(c)

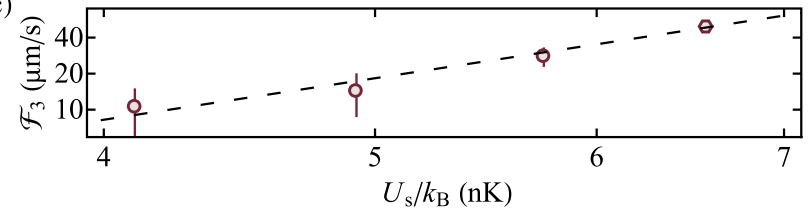

FIG. 4. Observation of third-harmonic generation. (a) Steadystate center-of-mass velocity response $v\left(t_{s}\right)$ for a strong drive $\left(U_{s} / k_{B}=6.6 \mathrm{nK}\right)$ at $\omega /(2 \pi)=9 \mathrm{~Hz}$ (top) and $\omega /(2 \pi)=$ $3.5 \mathrm{~Hz}$ (bottom). In the latter case, we clearly observe a nonmonochromatic response. The solid lines show fits to the data [55]. (b) Corresponding Fourier spectra $\mathcal{F}$, which for $\omega /(2 \pi)=$ $3.5 \mathrm{~Hz}$ reveals a response at $3 \omega$. (c) Extracted third-harmonic generation amplitude $\mathcal{F}_{3}$ for $\omega /(2 \pi)=3.5 \mathrm{~Hz}$ plotted as a function of $U_{s}$ on log-log scale. The dashed line shows a power-law fit, which gives $\mathcal{F}_{3} \propto U_{s}^{3.6(1.2)}$, consistent with the cubic scaling behavior characteristic of third-harmonic generation.

$\alpha /(L \sqrt{N})=2^{5 / 4} / \pi^{3 / 2} \sqrt{\xi / L}=0.091(4)$ [dashed line in Fig. 3(b)], which is close to the experimental value.

Furthermore, we find that $\Gamma \propto U_{s}^{0.67(2)}$, which, interestingly, suggests that even though $v_{m} / U_{s}$ and $\Gamma$ are not independent of $U_{s}$ (as they would be for a linearly damped harmonic oscillator), one still recovers $v_{m} \Gamma \propto U_{s}$. This reflects the energy balance condition for a driven-dissipative steady state; $U_{s} v_{m}$ is the driving power, and $v_{m}^{2} \Gamma$ is akin to the energy dissipation rate, the energy stored in the system being $\propto v_{m}^{2}$.

In Fig. 3(d), we show the complementary data on the phase of the velocity response, $\theta$, as a function of $U_{s}$ for various drive frequencies. As expected, $\theta$ is $\pi / 2(-\pi / 2)$ out of phase with the drive below (above) resonance. Interestingly, for low drive strengths $\left(U_{s} / k_{B}<1 \mathrm{nK}\right)$, the on-resonance $\theta\left(\omega_{d}\right)$ is observably nonzero and nearly independent of $U_{s}$. Averaging the data for $U_{s} / k_{B}<1 \mathrm{nK}$ gives $\theta=-0.12(4)$, which suggests that $\operatorname{Re}\left[\kappa_{2}\right]=$ $0.12(4) \operatorname{Im}\left[\kappa_{2}\right]$ within our model [see Sec. IV in [51]); this a posteriori justifies our initial assumption that $\left|\operatorname{Re}\left[\kappa_{2}\right] / \operatorname{Im}\left[\kappa_{2}\right]\right| \ll 1$. Including this small nonzero $\operatorname{Re}\left[\kappa_{2}\right]$ in the model further improves agreement with the data [dotted lines in Figs. 3(a) and 3(d)], but the difference is small.

For a nonlinear response, one also generically expects the possibility of harmonic generation. Experimentally, we did not observe evidence of higher-harmonic generation for $U_{s} / k_{B}<3 \mathrm{nK}$, so in the final part of this Letter, we turn to even larger drive amplitudes (up to $U_{s} / k_{B}=6.6 \mathrm{nK}$ ). For most driving frequencies $\omega$, we only observe a monochromatic response at the drive frequency, even for strong drives (see top panels in Figs. 4(a) and 4(b) where $\omega /(2 \pi)=9 \mathrm{~Hz}$ [53]). However, the behavior is markedly different for $\omega /(2 \pi)=3.5 \mathrm{~Hz}$ (bottom panels), for which $3 \omega$ is close to the resonant $\omega_{d}$. In this case, the Fourier spectrum of the time-domain response [Fig. 4(b)] shows a clear peak at $3 \omega$, signaling third-harmonic generation, in addition to the main peak at $\omega$ [54]. In Fig. 4(c), we plot the third-harmonic amplitude $\mathcal{F}_{3}$ versus $U_{s}$ on $\log -\log$ scale [55]. A power-law fit (dashed line) gives $\mathcal{F}_{3} \propto U_{s}^{3.6(1.2)}$, consistent with a cubic scaling in $U_{s}$ (characteristic of third-harmonic generation) and in qualitative agreement with predictions from Eq. (2) (Sec. V in [51]).

In conclusion, we have experimentally and theoretically studied the nonlinear decay of the fundamental gapped excitation of a Bose-Einstein condensate. Our experiments reveal a cubic damping mechanism as well as thirdharmonic generation, which we have shown can be captured by a mean-field model based on a microscopic theory at lowest order. The decay of lowest-lying gapped excitations is an understudied but generic problem in quantum many-body physics, and in the future, it would be interesting to transpose this study to other many-body systems such as the strongly interacting Fermi gas [56,57], dipolar gases [58-60], or topological systems [61]. We note that for our strongest (resonant) drive the gas is turbulent in steady state [49], and the many-body decay of the lowestlying mode provides a stepping stone toward the transfer of energy to higher-lying modes. Thus, our work paves the way for a microscopic understanding of the genesis of a turbulent cascade $[49,62]$, where the energy leakage from the driven discrete lowest-lying mode is sufficiently large to sustain a nonequilibrium steady state. Such a transition from the discrete-state dynamics to a continuum turbulent cascade has recently been theoretically studied in a cosmological context [63]. Finally, the exclusively nonlinear decay of the lowest-lying mode opens up the prospect of exploring quantum state preparation and the generation of nonclassical states of this degree of freedom, analogous to the quantum control of a single mode in optomechanics [64-66].

Data supporting this publication are available in the Apollo repository [67].

We thank Robert P. Smith, Maciej Gałka, and Nishant Dogra for helpful discussions and comments on the manuscript. This work was supported by EPSRC [Grants No. EP/N011759/1, No. EP/P009565/1, and No. EP/ K030094/1], ERC (QBox), QuantERA (NAQUAS, EPSRC Grant No. EP/R043396/1). N. N. acknowledges support from Trinity College (Cambridge), the David and Lucile Packard Foundation, the Alfred P. Sloan Foundation, and NSF CAREER (Grant No. 1945324). C.E. 
acknowledges support from Jesus College (Cambridge). T. A.H. acknowledges support from the EU Marie Skłodowska-Curie program [Grant No. MSCA-IF- 2018 840081]. Z.H. acknowledges support from the Royal Society Wolfson Fellowship. N. R. C. acknowledges the support of the Simons Foundation.

*These two authors contributed equally to this work. †ce330@cam.ac.uk zhengwei8796@foxmail.com

[1] D. Pines and P. Nozieres, Theory of Quantum Liquids (CRC Press, Boca Raton, FL, 2018).

[2] A. Griffin, Excitations in a Bose-Condensed Liquid (Cambridge University Press, Cambridge, England, 1993).

[3] A. L. Fetter and J. D. Walecka, Quantum Theory of ManyParticle Systems (Dover Publications, New York, 2003).

[4] F. Bloch, Über die Quantenmechanik der Elektronen in Kristallgittern, Z. Phys. 52, 555 (1929).

[5] L. Landau, Theory of the superfluidity of Helium II, Phys. Rev. 60, 356 (1941).

[6] L. N. Cooper, Bound electron pairs in a degenerate fermi gas, Phys. Rev. 104, 1189 (1956).

[7] W. Kohn, Theory of the insulating state, Phys. Rev. 133, A171 (1964).

[8] E. M. Henley and L. Wilets, Energy gap in nuclear matter. I. Extended theory, Phys. Rev. 133, B1118 (1964).

[9] R.C. Ashoori, Electrons in artificial atoms, Nature (London) 379, 413 (1996).

[10] P. G. Klemens, Anharmonic decay of optical phonons, Phys. Rev. 148, 845 (1966).

[11] B. L. Altshuler, Y. Gefen, A. Kamenev, and L. S. Levitov, Quasiparticle Lifetime in a Finite System: A Nonperturbative Approach, Phys. Rev. Lett. 78, 2803 (1997).

[12] A. Kolezhuk and S. Sachdev, Magnon Decay in Gapped Quantum Spin Systems, Phys. Rev. Lett. 96, 087203 (2006).

[13] A. L. Chernyshev, M. E. Zhitomirsky, N. Martin, and L.-P. Regnault, Lifetime of Gapped Excitations in a Collinear Quantum Antiferromagnet, Phys. Rev. Lett. 109, 097201 (2012).

[14] L. Landau and I. M. Khalatnikov, The theory of the viscosity of Helium II. I. Collisions of elementary excitations in Helium II, Zh. Eksp. Teor. Fiz. 19, 637 (1949).

[15] K. Bedell, D. Pines, and A. Zawadowski, Pseudopotential theory of interacting roton pairs in superfluid ${ }^{4} \mathrm{He}$, Phys. Rev. B 29, 102 (1984).

[16] K. H. Andersen, J. Bossy, J. C. Cook, O. G. Randl, and J.-L. Ragazzoni, High-Resolution Measurements of Rotons in ${ }^{4}$ He, Phys. Rev. Lett. 77, 4043 (1996).

[17] D. S. Jin, J. R. Ensher, M. R. Matthews, C. E. Wieman, and E. A. Cornell, Collective Excitations of a Bose-Einstein Condensate in a Dilute Gas, Phys. Rev. Lett. 77, 420 (1996).

[18] M.-O. Mewes, M. R. Andrews, N. J. van Druten, D. M. Kurn, D. S. Durfee, C. G. Townsend, and W. Ketterle, Collective Excitations of a Bose-Einstein Condensate in a Magnetic Trap, Phys. Rev. Lett. 77, 988 (1996).

[19] M. R. Andrews, D. M. Kurn, H.-J. Miesner, D. S. Durfee, C. G. Townsend, S. Inouye, and W. Ketterle, Propagation of
Sound in a Bose-Einstein Condensate, Phys. Rev. Lett. 79, 553 (1997).

[20] D. M. Stamper-Kurn, H.-J. Miesner, S. Inouye, M. R. Andrews, and W. Ketterle, Collisionless and Hydrodynamic Excitations of a Bose-Einstein Condensate, Phys. Rev. Lett. 81, 500 (1998).

[21] C. Raman, M. Köhl, R. Onofrio, D. S. Durfee, C. E. Kuklewicz, Z. Hadzibabic, and W. Ketterle, Evidence for a Critical Velocity in a Bose-Einstein Condensed Gas, Phys. Rev. Lett. 83, 2502 (1999).

[22] D. M. Stamper-Kurn, A. P. Chikkatur, A. Görlitz, S. Inouye, S. Gupta, D. E. Pritchard, and W. Ketterle, Excitation of Phonons in a Bose-Einstein Condensate by Light Scattering, Phys. Rev. Lett. 83, 2876 (1999).

[23] O. M. Maragò, S. A. Hopkins, J. Arlt, E. Hodby, G. Hechenblaikner, and C. J. Foot, Observation of the Scissors Mode and Evidence for Superfluidity of a Trapped Bose-Einstein Condensed Gas, Phys. Rev. Lett. 84, 2056 (2000).

[24] K. W. Madison, F. Chevy, W. Wohlleben, and J. Dalibard, Vortex Formation in a Stirred Bose-Einstein Condensate, Phys. Rev. Lett. 84, 806 (2000).

[25] J. R. Abo-Shaeer, C. Raman, J. M. Vogels, and W. Ketterle, Observation of vortex lattices in Bose-Einstein condensates, Science 292, 476 (2001).

[26] J. Steinhauer, R. Ozeri, N. Katz, and N. Davidson, Excitation Spectrum of a Bose-Einstein Condensate, Phys. Rev. Lett. 88, 120407 (2002).

[27] J. M. Vogels, K. Xu, C. Raman, J. R. Abo-Shaeer, and W. Ketterle, Experimental Observation of the Bogoliubov Transformation for a Bose-Einstein Condensed Gas, Phys. Rev. Lett. 88, 060402 (2002).

[28] N. Katz, J. Steinhauer, R. Ozeri, and N. Davidson, Beliaev Damping of Quasiparticles in a Bose-Einstein Condensate, Phys. Rev. Lett. 89, 220401 (2002).

[29] L. Khaykovich, F. Schreck, G. Ferrari, T. Bourdel, J. Cubizolles, L. D. Carr, Y. Castin, and C. Salomon, Formation of a matter-wave bright soliton, Science 296, 1290 (2002).

[30] K. E. Strecker, G. B. Partridge, A. G. Truscott, and R. G. Hulet, Formation and propagation of matter-wave soliton trains, Nature (London) 417, 150 (2002).

[31] I. Coddington, P. Engels, V. Schweikhard, and E. A. Cornell, Observation of Tkachenko Oscillations in Rapidly Rotating Bose-Einstein Condensates, Phys. Rev. Lett. 91, 100402 (2003).

[32] V. Bretin, P. Rosenbusch, F. Chevy, G. V. Shlyapnikov, and J. Dalibard, Quadrupole Oscillation of a Single-Vortex Bose-Einstein Condensate: Evidence for Kelvin Modes, Phys. Rev. Lett. 90, 100403 (2003).

[33] Z. Hadzibabic, P. Krüger, M. Cheneau, B. Battelier, and J. Dalibard, Berezinskii-Kosterlitz-Thouless crossover in a trapped atomic gas, Nature (London) 441, 1118 (2006).

[34] P. Engels, C. Atherton, and M. A. Hoefer, Observation of Faraday Waves in a Bose-Einstein Condensate, Phys. Rev. Lett. 98, 095301 (2007).

[35] S. B. Papp, J. M. Pino, R. J. Wild, S. Ronen, C. E. Wieman, D. S. Jin, and E. A. Cornell, Bragg Spectroscopy of a Strongly Interacting ${ }^{85} \mathrm{Rb}$ Bose-Einstein Condensate, Phys. Rev. Lett. 101, 135301 (2008). 
[36] R. Meppelink, S. B. Koller, and P. van der Straten, Sound propagation in a Bose-Einstein condensate at finite temperatures, Phys. Rev. A 80, 043605 (2009).

[37] J.-C. Jaskula, G. B. Partridge, M. Bonneau, R. Lopes, J. Ruaudel, D. Boiron, and C. I. Westbrook, Acoustic Analog to the Dynamical Casimir Effect in a Bose-Einstein Condensate, Phys. Rev. Lett. 109, 220401 (2012).

[38] R. Lopes, C. Eigen, A. Barker, K. G. H. Viebahn, M. Robert-de-Saint-Vincent, N. Navon, Z. Hadzibabic, and R. P. Smith, Quasiparticle Energy in a Strongly Interacting Homogeneous Bose-Einstein Condensate, Phys. Rev. Lett. 118, 210401 (2017).

[39] L. W. Clark, A. Gaj, L. Feng, and C. Chin, Collective emission of matter-wave jets from driven Bose-Einstein condensates, Nature (London) 551, 356 (2017).

[40] J. L. Ville, R. Saint-Jalm, É. Le Cerf, M. Aidelsburger, S. Nascimbène, J. Dalibard, and J. Beugnon, Sound Propagation in a Uniform Superfluid Two-Dimensional Bose Gas, Phys. Rev. Lett. 121, 145301 (2018).

[41] S. J. Garratt, C. Eigen, J. Zhang, P. Turzák, R. Lopes, R. P. Smith, Z. Hadzibabic, and N. Navon, From single-particle excitations to sound waves in a box-trapped atomic BoseEinstein condensate, Phys. Rev. A 99, 021601(R) (2019).

[42] L. Feng, J. Hu, L. W. Clark, and C. Chin, Correlations in high-harmonic generation of matter-wave jets revealed by pattern recognition, Science 363, 521 (2019).

[43] P. Christodoulou, M. Gałka, N. Dogra, R. Lopes, J. Schmitt, and Z. Hadzibabic, Observation of first and second sound in a Berezinskii-Kosterlitz-Thouless superfluid, arXiv:2008.06044.

[44] For related studies on excitations of ultracold gases in optical lattices, see, e.g., [45,46].

[45] C. Gross and I. Bloch, Quantum simulations with ultracold atoms in optical lattices, Science 357, 995 (2017).

[46] W. Hofstetter and T. Qin, Quantum simulation of strongly correlated condensed matter systems, J. Phys. B 51, 082001 (2018).

[47] N. N. Bogoliubov, On the theory of superfluidity, J. Phys. USSR 11, 23 (1947).

[48] S. T. Beliaev, Energy spectrum of a non-ideal Bose gas, Sov. Phys. JETP 7, 299 (1958).

[49] N. Navon, A. L. Gaunt, R. P. Smith, and Z. Hadzibabic, Emergence of a turbulent cascade in a quantum gas, Nature (London) 539, 72 (2016).

[50] A. L. Gaunt, T. F. Schmidutz, I. Gotlibovych, R. P. Smith, and Z. Hadzibabic, Bose-Einstein Condensation of Atoms in a Uniform Potential, Phys. Rev. Lett. 110, 200406 (2013).

[51] See Supplemental Material at http://link.aps.org/ supplemental/10.1103/PhysRevLett.126.060402 for theoretical details.

[52] More generally, a $M$-body process of this form would lead to a $1 /(2 M-1)$ power-law scaling (see Sec. VII in [51]).
[53] In this case, the response amplitude still follows the resonant scaling $v_{m} \propto U_{s}^{1 / 3}$ seen in Fig. 2(b).

[54] Note that the resonance condition for third-harmonic generation, $3 \omega=\omega_{d}$, is not stringent because of the strong nonlinear broadening; indeed, for our strongest drive, we observe a weak third-harmonic generation signal even for $\omega /(2 \pi)=5 \mathrm{~Hz}$.

[55] We extract $\mathcal{F}_{3}$ by fitting the data with $v\left(t_{s}\right)=$ $\mathcal{F}_{1} \sin \left(\omega t_{s}+\theta_{1}\right)+\mathcal{F}_{3} \sin \left(3 \omega t_{s}+\theta_{3}\right)$.

[56] P. B. Patel, Z. Yan, B. Mukherjee, R. J. Fletcher, J. Struck, and M. W. Zwierlein, Universal sound diffusion in a strongly interacting Fermi gas, Science 370, 1222 (2020).

[57] M. Bohlen, L. Sobirey, N. Luick, H. Biss, T. Enss, T. Lompe, and H. Moritz, Sound Propagation and QuantumLimited Damping in a Two-Dimensional Fermi Gas, Phys. Rev. Lett. 124, 240403 (2020).

[58] L. Tanzi, S. M. Roccuzzo, E. Lucioni, F. Famà, A. Fioretti, C. Gabbanini, G. Modugno, A. Recati, and S. Stringari, Supersolid symmetry breaking from compressional oscillations in a dipolar quantum gas, Nature (London) 574, 382 (2019).

[59] M. Guo, F. Böttcher, J. Hertkorn, J.-N. Schmidt, M. Wenzel, H. P. Büchler, T. Langen, and T. Pfau, The low-energy Goldstone mode in a trapped dipolar supersolid, Nature (London) 574, 386 (2019).

[60] L. Chomaz, D. Petter, P. Ilzhöfer, G. Natale, A. Trautmann, C. Politi, G. Durastante, R. M. W. van Bijnen, A. Patscheider, M. Sohmen, M. J. Mark, and F. Ferlaino, LongLived and Transient Supersolid Behaviors in Dipolar Quantum Gases, Phys. Rev. X 9, 021012 (2019).

[61] M.Z. Hasan and C.L. Kane, Colloquium: Topological insulators, Rev. Mod. Phys. 82, 3045 (2010).

[62] N. Navon, C. Eigen, J. Zhang, R. Lopes, A. L. Gaunt, K. Fujimoto, M. Tsubota, R. P. Smith, and Z. Hadzibabic, Synthetic dissipation and cascade fluxes in a turbulent quantum gas, Science 366, 382 (2019).

[63] A. Chatrchyan, K. Geier, M. K. Oberthaler, J. Berges, and P. Hauke, Analog reheating of the early universe in the laboratory, arxiv:2008.02290.

[64] L. Gilles and P. L. Knight, Two-photon absorption and nonclassical states of light, Phys. Rev. A 48, 1582 (1993).

[65] H. Tan, F. Bariani, G. Li, and P. Meystre, Generation of macroscopic quantum superpositions of optomechanical oscillators by dissipation, Phys. Rev. A 88, 023817 (2013).

[66] M. Aspelmeyer, T. J. Kippenberg, and F. Marquardt, Cavity optomechanics, Rev. Mod. Phys. 86, 1391 (2014).

[67] https://doi.org/10.17863/CAM.63897. 